\title{
Planification et animation de trois types d'ateliers pédagogiques
}

\author{
How to plan and facilitate three types of educational workshops?
}

\author{
Gilles GIRARD, Diane CLAVET, Richard BOULÉ
}

\begin{abstract}
Messages clés - Les auteurs distinguent trois types d'ateliers pédagogiques en fonction de leurs cibles respectives : a) l'atelier de production a comme cible une réalisation commune; b) l'atelier de formation, type apprentissage, vise l'acquisition d'habiletés et c) l'atelier de formation, type exploration, a comme objectif des prises de conscience ou des changements d'attitudes. Les adultes apprennent de façon plus efficace lorsquills identifient, explicitement et d'emblée, l'utilité, à court terme, de ce quills ont à acquérir ou la tâche précise quills pourront mieux accomplir au terme de leur apprentissage. $\bullet$ Le corps de l'atelier de formation consiste à faire vivre aux participants une expérience pertinente, en lien avec les apprentissages ou les changements visés, au moyen d'exercices minutieusement planifiés. - Cette expérimentation basée sur la pratique doit sassocier à la réflexion pendant et après l'action pour permettre une compréhension et une éventuelle modification des schèmes conceptuels préexistants et, ultérieurement, un changement de pratique. $\bullet$ Le déroulement de l'atelier doit allouer aux participants plus de temps de travail actif à résoudre des problèmes et à générer des solutions que de temps d'écoute des animateurs; ceux-ci aident le groupe en partageant brièvement leurs expériences professionnelles en tant que modèles de rôle. - La planification d'un atelier par des novices dans le domaine requiert le soutien d'un consultant expert en conception d'ateliers. Cette démarche guidée est une excellente activité de formation professorale pour le planificateur de l'atelier.
\end{abstract}

Mots clés Atelier pédagogique ; animation de groupe ; éducation médicale.

Key messages • The authors distinguish three types of educational workshops according to their respective goals: $A$ ) the production workshop aims at a common realization; B) the learning workshop, as training, aims at developing skills; $C$ ) the learning workshop, as experimentation, aims at awareness or change of attitudes. - Adults learn more efficiently when informed from the start of the short term usefulness of what they need to acquire or what specific task they will be able to better accomplish at the end of the learning. - The main part of the learning workshop allows the participants a significant experience linked to the skills or changes aimed through meticulously planned exercises. $\bullet$ This real practice-based experimentation must be associated to a reflexion during and after the action to allow an understanding and a possible modification of the pre-existing conceptual frameworks and, ultimately, a change in practice. - The planning of the workshop sequence must allow more work time for the participants in solving problems and generating solutions rather than listening to the facilitators; they help the group in briefly exchanging their professional experiences as role models. $\bullet$ To plan a workshop, beginners in the field require the support of an expert consultant in workshop's designing. Working with a consultant is an excellent faculty development opportunity for the workshop planner.

Key words Educational workshop; group facilitation; medical education.

Pédagogie Médicale 2008;9:42-53

1- Département de médecine de famille, Faculté de médecine, Université de Sherbrooke.

Correspondance : Gilles Girard - Département de médecine de famille - Faculté de médecine - Université de Sherbrooke - $300112^{\circ}$

Avenue Nord, Sherbrooke - Québec Canada J1H 5N4. Téléphone : (819) 346-1110, extension 14273 - Télécopieur : (819) $564-5386$.

Mailto:ggirard.csss-iugs@ssss.gouv.qc.ca 


\section{Planification et animation de trois types d'ateliers pédagogiques}

\section{Introduction}

Qu'il est frustrant de s'inscrire à un atelier et de découvrir sur place qu'en réalité, il s'agit plutôt d'un exposé interactif ! Mais qu'est-ce qui distingue un atelier d'une présentation interactive ? Jean ${ }^{1}$ définit un atelier comme une rencontre qui permet à des personnes d'échanger entre elles et avec des experts, dans un climat de coopération, dans le but d'acquérir de nouvelles connaissances, habiletés ou attitudes sur des sujets ou des problèmes d'intérêt commun. C'est donc une séance qui engage activement les participants dans des échanges en vue d'une production commune ou pour les assister dans l'acquisition de compétences et/ou de changements d'attitudes professionnelles. Cela differe d'une présentation qui, bien qu'elle doive idéalement maintenir les auditeurs actifs et engagés cognitivement pour apprendre, n'offre que peu d'interactions entre les participants et privilégie l'apprentissage de connaissances déclaratives.

Le propos de cet article est d'illustrer la planification et le déroulement opérationnel d'un atelier à partir d'une revue de la littérature et de l'expérience des auteurs dans la préparation et l'animation d'ateliers, ainsi que lors du coaching d'animateurs d'ateliers dans divers contextes tels que la formation des résidents en médecine, la formation médicale continue et la formation pédagogique des enseignants en médecine. Après avoir défini trois types d'ateliers, les auteurs décrivent la planification et le déroulement d'un atelier dans le but de fournir des balises pour sa préparation. Son déroulement est présenté sous la forme d'une grille de contrôle de qualité à l'intention de l'animateur d'atelier.

\section{Un atelier? A certaines conditions...}

Un atelier pédagogique est une activité exigeante pour les animateurs et les participants, d'où l'importance d'une planification rigoureuse et soignée ${ }^{1,2}$ de la part des animateurs et d'une décision libre et éclairée des participants de s'engager activement dans l'atelier. Les auteurs distinguent trois types d'ateliers (tableau 1) en fonction de leurs cibles et de leurs finalités respectives. D'une part, les ateliers de production regroupent des experts ou des participants concernés par une situation donnée, qui s'entendent pour effectuer une tâche en groupe de type résolution de problème. Ils aboutissent à une production commune générée au moyen d'une procédure reconnue guidée par les animateurs. D'autre part, les ateliers de formation sont de deux types : les ateliers d'apprentissage et les ateliers d'exploration. Les ateliers d'apprentissage comportent la description ou idéalement la démonstration d'une habileté et une initiation à la pratique de cette habileté avec feedback sur la performance à l'aide d'une grille d'observation détaillée. Les ateliers d'exploration mettent l'accent sur une démarche réflexive conjointe des participants à partir d'une expérience significative reliée à des dimensions ciblées de leur vécu professionnel. Notons que, dans une séance de travail d'une journée par exemple, une période de temps peut être consacrée à un atelier de type production alors qu'une autre vise un apprentissage spécifique. De fait, il est possible de combiner ces différents types d'ateliers au cours d'une activité de formation.

Chaque type d'ateliers appelle un déroulement et des méthodes pédagogiques spécifiques. Sous la rubrique "Déroulement typique " du tableau 1, un guide générique est proposé pour chaque type d'atelier ; il constitue en quelque sorte la recette à suivre pour garder le cap sur la cible et utiliser les méthodes pédagogiques appropriées pour ce faire. Le tableau 2 fournit une illustration pour chaque type d'atelier : le premier exemple porte sur l'identification des difficultés rencontrées par des résidents de médecine de famille au cours de leur formation et les solutions qu'ils proposent ${ }^{3}$, le deuxième sur l'annonce d'une mauvaise nouvelle ${ }^{4,5}$ et le troisième sur l'intimité dans la relation patient-médecin ${ }^{6}$. La séquence spécifique prévue lors de ces ateliers y est décrite et traduit concrètement le déroulement typique proposé au tableau précédent.

Les principes qui vont suivre s'appliquent aux trois types d'ateliers, sauf pour quelques particularités qui seront notées. Idéalement, le nombre de participants à un atelier se situe entre six et douze ${ }^{9}$ de manière à faciliter à la fois une participation active de chacun et des interactions efficaces par rapport aux objectifs visés. Lorsque le nombre de participants à un atelier d'apprentissage est supérieur à douze, il faut prévoir des ajustements de fonctionnement, qui seront présentés plus loin, et limiter l'ampleur des objectifs visés : par exemple, parler davantage de sensibilisation à une thématique que de maîtrise d'une compétence puisque les opportunités de pratique avec feedback sont restreintes. De plus, le temps prévu pour un atelier, habituellement entre quatre-vingt-dix minutes et trois heures, doit être suffisant pour vivre et discuter une expérience pertinente qui permette d'atteindre les objectifs de l'atelier. Pour l'acquisition de compétences complexes, on prévoit des ateliers d'une ou deux journées de six heures ou 


\section{Références}

même une séquence de quelques ateliers s'échelonnant dans le temps avec des tâches individuelles entre les ateliers, par exemple : un programme de cinq ateliers de trois heures sur la tâche du superviseur en supervision directe avec quinze heures de travaux individuels préparatoires et d'intégration réparties entre les ateliers.

\begin{tabular}{|c|c|c|c|}
\hline \multicolumn{4}{|c|}{$\begin{array}{c}\text { Tableau 1 : } \\
\text { Types d'ateliers pédagogiques }\end{array}$} \\
\hline Types d'ateliers & A) Production & $\begin{array}{l}\text { B) Formation : } \\
\text { Type apprentissage }\end{array}$ & $\begin{array}{l}\text { C) Formation : } \\
\text { Type exploration }\end{array}$ \\
\hline Objectifs & $\begin{array}{l}\text { Consensus sur une problé- } \\
\text { matique, élaboration d'un } \\
\text { outil pédagogique, fabrica- } \\
\text { tion de matériel ou de } \\
\text { documentation issue de } \\
\text { l'expérience. }\end{array}$ & $\begin{array}{l}\text { Maîtrise d'une habileté ou } \\
\text { compétence. }\end{array}$ & $\begin{array}{l}\text { Prise de conscience et modi- } \\
\text { fication des attitudes }\end{array}$ \\
\hline Exemples & $\begin{array}{l}\text { Difficultés et solutions au } \\
\text { cours de la résidence en } \\
\text { médecine de famille : } \\
\text { outil d'autoréflexion }^{3} \text {. }\end{array}$ & $\begin{array}{l}\text { Comment annoncer une } \\
\text { mauvaise nouvelle ?4,5 }\end{array}$ & $\begin{array}{l}\text { Intimité dans la relation } \\
\text { patient-médecin }: \text { vos } \\
\text { attitudes professionnelles ?6 }\end{array}$ \\
\hline $\begin{array}{l}\text { Niveau d'implication } \\
\text { des participants }\end{array}$ & Modéré & Elevé & Très élevé \\
\hline Déroulement typique & $\begin{array}{l}\text { - Préciser la pertinence de la } \\
\text { problématique à traiter, } \\
\text { le type de production } \\
\text { souhaité et son utilité. } \\
\text { - Appliquer une procédure } \\
\text { adaptée à la genèse du } \\
\text { type de contenu visé. } \\
\text { - Procéder à une synthèse et } \\
\text { à une validation du } \\
\text { contenu produit (et/ou } \\
\text { planifier une validation } \\
\text { ultérieure à plus large } \\
\text { échelle). }\end{array}$ & $\begin{array}{l}\text { - Démonstration axée sur } \\
\text { les aspects difficiles de } \\
\text { l'habileté à maîtriser ou } \\
\text { description détaillée de ce } \\
\text { qu'il faudra pratiquer, } \\
\text { - Pratique avec feedback, } \\
\text { ex. : jeu de rôle, pratique } \\
\text { en trio, } \\
\text { - Retour sur le processus et/ } \\
\text { ou sur les éléments qui } \\
\text { demeurent difficiles à } \\
\text { maîtriser (prévoir un nou- } \\
\text { vel exercice d'apprentis- } \\
\text { sage si nécessaire). }\end{array}$ & $\begin{array}{l}\text { - Bref exercice d'introspec- } \\
\text { tion (une question, réac- } \\
\text { tion à une vidéo) permet- } \\
\text { tant aux participants de } \\
\text { prendre conscience de leur } \\
\text { position sur le sujet. } \\
\text { - Mises en situation stimulant } \\
\text { l'exploration de positions } \\
\text { variées travaillées en sous- } \\
\text { groupes, en alternant action } \\
\text { et réflexion sur l'action. } \\
\text { - Discussion-synthèse permet- } \\
\text { tant aux participants de } \\
\text { dégager de l'exploration « un } \\
\text { pas de plus " par rapport à } \\
\text { leur position initiale. }\end{array}$ \\
\hline $\begin{array}{l}\text { Particularités } \\
\text { concernant } \\
\text { l'animation }\end{array}$ & $\begin{array}{l}\text { Respecte les conditions } \\
\text { d'application de la procé- } \\
\text { dure choisie : ex. entretien } \\
\text { focalisé (focus group } \\
\text { groupe nominal'. }\end{array}$ & $\begin{array}{l}\text { Suppose une expertise de } \\
\text { contenu et une compétence } \\
\text { dans l'animation d'exercices } \\
\text { d'apprentissage (ex. : jeux } \\
\text { de rôle, pratique en trio). }\end{array}$ & $\begin{array}{l}\text { Suppose une maîtrise de } \\
\text { l'approche réflexive et de } \\
\text { stratégies pour la guider en } \\
\text { groupe, incluant la gestion } \\
\text { des aspects émotifs. }\end{array}$ \\
\hline
\end{tabular}




\section{Tableau 2 : \\ Exemples de plans des trois types d'ateliers}

\section{A) Atelier de type "production " : Difficultés et solutions au cours de la résidence}

B) Atelier de formation de type " apprentissage" : nouvelle

1. Introduction : vos expériences

1. Objectifs du travail : élaborer un outil d'autoréflexion sur le thème à l'intention des résidents.

2. Technique du groupe nominal : "Qu'est-ce qui vous empêche de fonctionner à votre goût comme résident(e) ? » et " Quelles stratégies peuvent vous aider à composer avec les difficultés liées à la vie de résident(e) ?"

3. Echange sur les usages potentiels de l'information produite.

4. Synthèse par les animateurs et validation par le groupe.

5. Conclusion et évaluation de l'atelier. nouvelles?

2. Exercice «Vos conseils à votre collègue sur le point d'annoncer une mauvaise nouvelle?".

3. Description de l'habileté à acquérir sous forme de grille d'entrevue.

4. Pratique en trio et retour en faisant le lien avec des situations de leur pratique.

5. Jeu de rôle collectif : situation de déni. Annonce d'une mauvaise

B) Atelier de formation de type " exploration» : Intimité dans la relation patient-médecin

1. Introduction : vos réponses au pré-test : "Inconfort à dire non dans diverses situations? »

6. Discussion synthèse : autres applications à partir des incidents critiques rédigés préalablement à l'atelier (ex. : annonce du décès d'un jeune à l'urgence).

7. Conclusion et évaluation de l'atelier.
2. Jeux de rôle collectifs suivis de discussion en groupe des réponses évoquées et de leurs implications sur les thèmes suivants :

a) invitations et cadeaux des patients,

b) demandes de traitement d'un proche : "que faites-vous ? impacts de vos décisions? ", c) " à quelles conditions un contact physique avec un patient est-il respectueux ?"

3. Discussion synthèse : signaux d'éveil dans la relation avec un patient.

4. Conclusion et évaluation de l'atelier. 


\section{Références}

Nos expériences antérieures en tant que participants ou animateurs d'ateliers influencent grandement nos choix ultérieurs et la qualité de notre implication. Pour tirer profit de vos expériences en vue de la préparation d'un prochain atelier ${ }^{10}$, prenez quelques instants pour réfléchir à un ou deux incidents malheureux dont vous avez été témoin et qui ont empêché qu'une telle activité réponde à vos attentes. Plusieurs des erreurs courantes sont énoncées au tableau 3 que nous vous invitons à lire attentivement dès maintenant. Il décrit aussi les conditions de succès d'un atelier à chacune des étapes de son déroulement. Cela introduira la lecture des trois prochaines parties qui présentent les façons suivantes de planifier un atelier pour prévenir ce type d'erreurs : assurer une cohérence entre les objectifs-besoins-messages, proposer des exercices clairs, stimulants et structurer une animation efficace.

\section{Objectifs - Besoins - Messages}

Par où commencer lorsqu'on veut élaborer un atelier de formation ? Pensez à un thème d'atelier à bâtir et répondez aux trois questions suivantes :

- Que souhaitez-vous que les participants fassent différemment après l'atelier?

- Quelles pourraient être leurs réticences à adopter cette nouvelle façon de faire (croyances, contexte défavorisant, etc.) ?

- Identifiez ce qui pourrait les motiver à procéder à ces changements même si cela nécessite un effort.

Les adultes apprennent de façon plus efficace lorsqu'ils identifient, explicitement et d'emblée, l'utilité, à court terme, de ce qu'ils ont à acquérir ou la tâche précise qu'ils pourront mieux accomplir au terme de leur apprentissage ${ }^{1,2}$. A partir des réponses à ces questions, formulez une première ébauche des objectifs généraux et spécifiques de l'atelier. Ces objectifs doivent être réalistes, observables, orientés vers l'action et spécifiques en termes de changements visés. Voici un exemple tiré d'un atelier sur la communication patient-médecin : à la fin de l'atelier, les participants se seront exercés à interrompre un patient de façon efficace et respectueuse dans la perspective de résoudre efficacement son problème de santé. Les objectifs doivent aussi être adaptés à la durée de l'atelier, au nombre, à la préparation et à l'expertise des participants par rapport au contenu de l'atelier. On doit aussi tenir compte de l'homogénéité ou de l'hétérogénéité du groupe, spécialement dans les ateliers d'apprentissage qui fonctionnent mieux lorsque le groupe est homogène en termes de pré-requis par rapport aux objectifs visés. Par exemple, dans un jeu de rôle sur l'art du feedback, un novice apprendra à s'enquérir de la perception de l'apprenant pour ensuite ajouter ce qu'il a observé et s'entendre avec lui sur les faits. Le participant expérimenté effectuera rapidement ces premières étapes et pourra se consacrer davantage à explorer et à valider des hypothèses expliquant l'écart entre les comportements observés et souhaités.

\section{Préciser les besoins : deux fois sur le métier...}

Bien qu'en pratique, un projet d'atelier de formation naisse souvent dans la tête des animateurs, il importe de s'enquérir rapidement des besoins des participants pour ajuster les objectifs à leurs réalités et préciser les messages ou les apprentissages visés. Chamberland et Hivon ${ }^{11}$ soulignent qu'un atelier de formation doit être centré à la fois sur les participants et sur leur contexte de pratique professionnelle et d'enseignement. On doit tenir compte des connaissances antérieures des participants, de leurs croyances et expertises et les placer en situation de résolution de problèmes réels cliniques et pédagogiques. L'engagement dans la réalisation de tâches professionnelles authentiques constitue le contexte d'apprentissage le plus motivant pour l'apprenant et le plus puissant pour l'enseignement et l'apprentissage des compétences intégrées au service de la résolution de problèmes de santé des patients ${ }^{11}$. Des techniques d'investigation complémentaires, telles que l'incident critique ${ }^{12}$, le groupe nominal ${ }^{7}$ et l'entretien focalisé8 (focus group), sont utiles pour cerner les besoins réels de formation des participants. Ces techniques peuvent aussi servir à générer des données dans un atelier de production auprès d'un groupe d'apprenants et/ ou d'experts. En outre, Gutnik et Gutnik ${ }^{2}$ ont développé dans leur ouvrage diverses fiches pratiques utiles à consulter aux diverses étapes de la préparation et de l'animation de formations.

Lors de l'atelier, l'engagement des participants est facilité s'ils sont informés qu'on a le souci de répondre à leurs besoins réels qu'on a pris soin de documenter. Pourtant, l'une des erreurs les plus fréquentes est de négliger de répondre à leurs besoins concrets ou de ne pas tenir compte de leurs connaissances, compétences et attitudes préalables à l'atelier. De plus, à divers moments de l'élaboration d'un atelier, il est utile de le soumettre à la critique d'éventuels participants pour conserver le cap sur leurs besoins et leur réalité. Au début de l'atelier, le fait d'annoncer et de situer les objectifs et le déroulement de l'atelier par rapport aux attentes du groupe (besoins ressentis) permet de développer un agenda commun et encourage la cohésion du groupe. 


\section{Planification et animation de trois types d'ateliers pédagogiques}

\section{Grille de contrôle de qualité pour les animateurs d'ateliers}

\begin{tabular}{|l|}
\hline \multicolumn{1}{|c|}{ Comportements souhaités } \\
\hline COMMENCER L'ATELIER DU BON PIED : \\
- Introduire le thème de l'atelier en illustrant sa perti- \\
nence par rapport à la réalité des participants (évo- \\
quer par exemple les questions auxquelles il vise à \\
répondre ou ce qu'ils sauront faire différemment \\
suite à cette activité de formation).
\end{tabular}

- Présenter les animateurs en soulignant leur expertise par rapport au thème et utiliser une méthode efficace pour connaître "qui sont les participants" (leur expérience en lien avec le thème).

- Enoncer les objectifs principaux de l'atelier en mettant en parallèle un aperçu du déroulement de l'atelier et ce qu'on attend des participants.

- Mettre le groupe en train: faire réfléchir les participants par rapport au thème (ex. : courte réflexion en lien avec leur pratique) et sur leurs besoins (lacunes à combler).

\section{CORPS DE L'ATELIER : FAIRE VIVRE UNE EXPÉRIENCE}

- Gérer le temps planifié pour chacune des sections et activités de l'atelier tout en tenant compte des besoins des participants.

- Présenter le contenu à apprendre sous la forme d'un schéma de référence qui aidera les participants à s'engager dans les différences expériences de l'atelier.

- Amener les participants à vivre une expérience en lien avec les apprentissages visés (messages) en équilibrant le temps de travail en sous-groupes et en plénière, surtout lorsque le nombre de participants est supérieur à 10 . a) donner des consignes claires et détaillées pour les tâches concrètes à effectuer ;

b) à partir des réponses anticipées, préparer le cadre de recueil des données en plénière afin de faciliter leur synthèse pour soutenir la progression de l'atelier ;

c) effectuer un retour sur l'exercice en groupe de façon stimulante et en évitant les redondances ;

d) formuler une synthèse qui intègre les éléments du retour sur la tâche et qui met en évidence les messages planifiés.
Pas un mot sur la pertinence du thème ; exemples non appropriés ou manquant de variété pour les divers participants.

Avec un nombre de participants $>15$, demander à chacun de se présenter individuellement. Suggestion : repérer à main levée les sous-groupes avec une expérience ou une caractéristique commune qu'il est utile de connaître pour l'animation (ex. : nouveaux enseignants).

Faire formuler les attentes de chacun. Suggestion : demander plutôt si certains ont des attentes particulières en plus des objectifs planifiés et préciser si, oui ou non, elles seront abordées.

Demander d'effectuer des tâches dont le processus ou les résultats ne seront pas utilisés dans la suite de l'atelier.

Effectuer une longue présentation théorique sans lien avec les tâches à effectuer.

Donner des tâches trop longues ou ambiguës qui ne sont ni significatives par rapport à la pratique des participants, ni directement pertinentes par rapport aux objectifs de l'atelier.

Aucune anticipation des résultats attendus et des façons de les gérer.

Faire répéter en plénière tout ce qui a été dit dans les sous-groupes de travail. Suggestion : cibler plutôt un élément clé par sous-groupe ou ce qui a été plus difficile à réaliser. 


\section{Références}

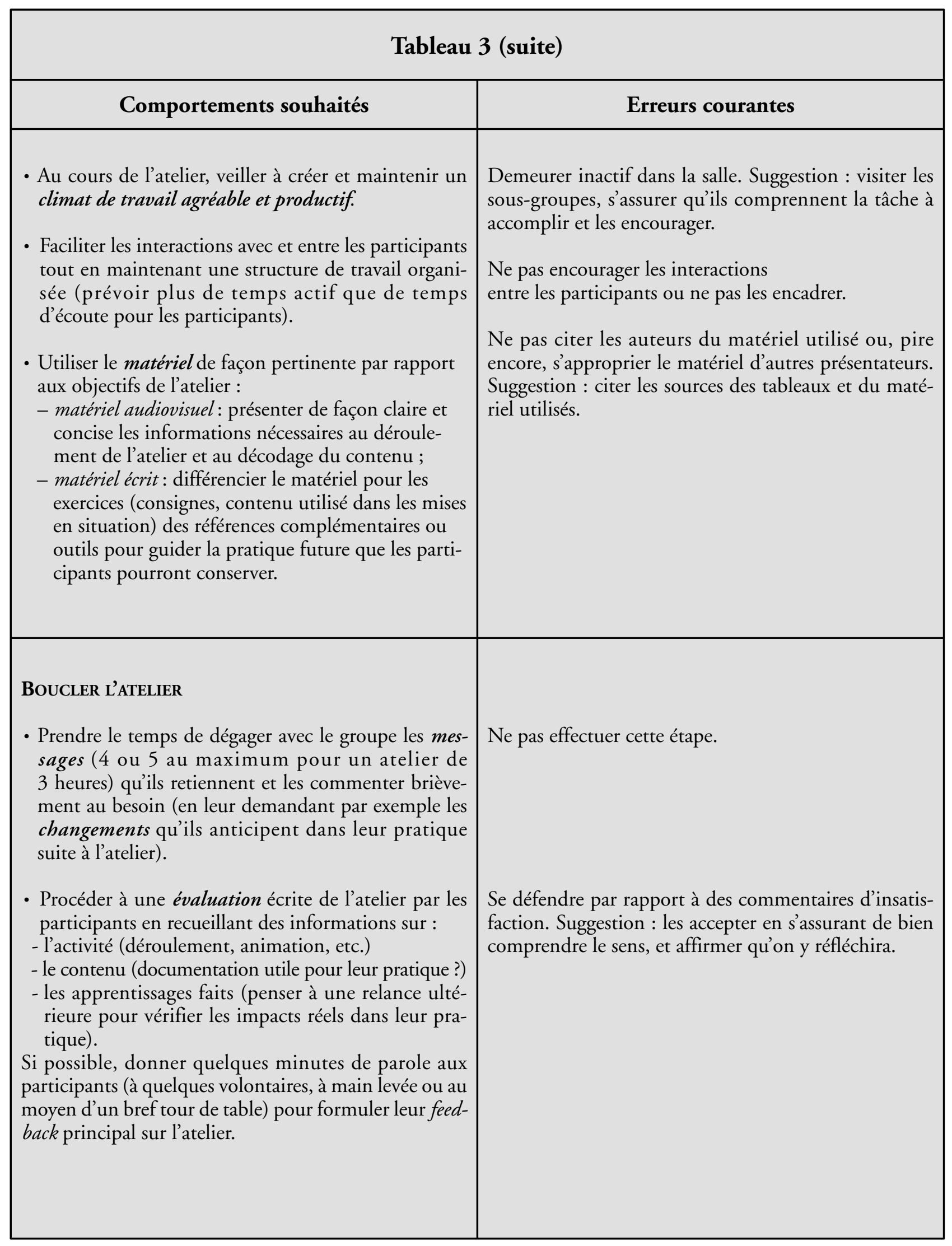




\section{Planification et animation de trois types d'ateliers pédagogiques}

Les animateurs se doivent d'être souples dans l'adaptation des scénarios d'un atelier lorsque l'auditoire requiert des ajustements par rapport aux attentes initiales ou au niveau de compétence visé, tout en préservant au mieux la cible initiale.

\section{Des exercices stimulants}

Le corps de l'atelier de formation consiste à réaliser une tâche commune ou à faire vivre une expérience pertinente aux participants, qui les amènent vers les apprentissages ou changements visés à travers une réflexion sur leur démarche ${ }^{13}$. Il s'agit d'un apprentissage dans et par l'action. Il importe toutefois d'éviter de répéter les étapes de la démarche que la plupart des participants maîtrisent déjà pour plutôt centrer l'exercice sur le passage clé vécu comme difficile ou source d'erreurs ou d'oublis fréquents. En regard de chacun de ces objectifs spécifiques, il importe de définir la tâche demandée aux participants, incluant les étapes à prévoir lorsque la tâche est complexe, et de donner des consignes claires pour la tâche ainsi que des informations sur le déroulement de la plénière ou de la phase d'intégration de l'expérience. Le défi consiste ici à choisir les activités ou les outils pédagogiques les plus appropriés pour remplir les objectifs visés. Ces outils impliquent un engagement du groupe dans un apprentissage actif, privilégiant la résolution de problèmes en lien évident avec leur contexte de travail (tableau 4). À cet égard, les pratiques en trios ${ }^{14}$ où les participants jouent alternativement les rôles $\mathrm{du}$ patient, du médecin et de l'observateur, sont très appréciés dans les ateliers d'apprentissage (outil guide en encadré). La technique de l'incident critique, utilisée avant un atelier de formation, permet de construire des exercices sur mesure (ex : jeux de rôle) en fonction des besoins et du niveau d'expertise des participants à partir des exemples réels de leur pratique qui y sont rapportés. De plus, il est recommandé d'opter pour des activités brèves (d'une durée maximale de 20 minutes) et de les varier pour maintenir l'intérêt et l'attention des participants.

La salle sera disposée en fonction des activités choisies et du nombre prévu de participants. Les tables en U facilitent les interactions entre les participants dans un petit groupe de six à douze participants, permettant à chacun d'avoir un contact visuel avec les autres. Avec un groupe plus nombreux, regrouper les participants autour de tables rondes de cinq à huit participants permet de planifier des activités en sous-groupes au sein desquels chaque participant demeure actif et bénéficie d'une interaction étroite avec quelques collègues. En présence de plusieurs îlots de travail, les animateurs fournissent des consignes plus détaillées qui permettront aux participants de recevoir le feedback formatif pertinent à travers l'exercice lui-même. Par exemple, dans une pratique en trio, le rôle du médecin comprendra les clés à essayer pour dénouer l'impasse. Le rôle du patient décrira des réactions à adopter selon le comportement du médecin. L'observateur, quant à lui, disposera, en plus de la grille d'observation, des clés requises pour conseiller celui qui joue le rôle du médecin. Au niveau du matériel audiovisuel, il importe de se limiter aux informations pertinentes et nécessaires à l'exécution et au décodage des tâches proposées et ainsi privilégier les interactions avec les participants. Lorsque certains éléments de contenu de type "messages à retenir pour la pratique " sont présentés lors de synthèses, les participants apprécient d'en avoir une copie, de même que quelques références complémentaires utiles au quotidien.

\section{Animation de l'atelier : plus facile lorsque l'atelier est bien structuré}

Même si les exercices proposés aux participants sont significatifs et stimulants, la pratique seule, bien que nécessaire, n'est pas suffisante et doit s'associer à la réflexion pendant et après l'action ${ }^{15}$ pour permettre une compréhension et une éventuelle modification des schèmes conceptuels préexistants. Dans une étude rétrospective portant sur dix ans, Boulé et $a l .{ }^{16}$ mettent en évidence l'importance de trois catégories de stratégies d'animation de groupe pour l'apprentissage des habiletés de communication patient-médecin-famille chez des résidents en médecine de famille : l'expérimentation concrète de nouvelles habiletés à l'aide de mises en situation en lien étroit avec leurs activités professionnelles (contextualisation), l'habitude de l'analyse réflexive dans et après l'action par des retours périodiques sur leur fonctionnement (décontextualiser pour dégager les principes sous-jacents) et la préparation au transfert des apprentissages par la discussion des ajustements à envisager lors des applications des acquis dans la pratique future (recontextualisation). Chamberland et Hivon ${ }^{11}$ notent également la place centrale de l'expérience pratique dans le développement des compétences. La triade de contextualisation, décontextualisation (ou abstraction) et recontextualisation est à la base du transfert des connaissances ${ }^{17}$.

Lors de l'élaboration des exercices, il est très utile d'anticiper les réactions et réponses possibles des participants pour aider, dans le feu de l'action au moment de l'atelier, 


\section{Références}

\begin{tabular}{|c|c|c|c|}
\hline \multicolumn{4}{|c|}{$\begin{array}{l}\text { Tableau } 4 \text { : } \\
\text { Outils pédagogiques et leur utilisation en atelier }\end{array}$} \\
\hline Outils & $\begin{array}{l}\text { Niveau d'activité } \\
\text { du participant }\end{array}$ & $\begin{array}{c}\text { Utilité } \\
\text { (et dans quels types d'ateliers) }\end{array}$ & Précautions à prendre \\
\hline $\begin{array}{l}\text { Exposés interactifs** } \\
\text { (quelques brèves capsules } \\
\text { de } 5-10 \text { minutes) }\end{array}$ & Faible & $\begin{array}{l}\text { Présenter les notions ciblées } \\
\text { ou les messages clés de l'atelier } \\
\left(\mathrm{A}, \mathrm{B}, \mathrm{C}^{*}\right) \text {. }\end{array}$ & $\begin{array}{l}\text { Les organiser sous forme de } \\
\text { schémas ou d'outils utilisables } \\
\text { en pratique. } \\
\text { Répondre aux questions de cla- } \\
\text { rification. }\end{array}$ \\
\hline $\begin{array}{l}\text { Exercice individuel écrit, } \\
\text { une étape préparatoire à } \\
\text { un échange ultérieur }\end{array}$ & Elevé & $\begin{array}{l}\text { Impliquer, susciter la réflexion } \\
\text { des participants en lien avec les } \\
\text { objectifs }(A, B, C) \text {. }\end{array}$ & $\begin{array}{l}\text { Consignes claires. Durée maxi- } \\
\text { male : } 5 \text { minutes. Prévoir son } \\
\text { utilisation. }\end{array}$ \\
\hline Discussion en dyades & Elevé & $\begin{array}{l}\text { Initier et personnaliser } \\
\text { les échanges. Efficace pour } \\
\text { mobiliser un groupe silencieux } \\
(\mathrm{A}, \mathrm{B}, \mathrm{C}) \text {. }\end{array}$ & $\begin{array}{l}\text { Éviter de demander les réponses } \\
\text { de chaque dyade en plénière } \\
\text { pour limiter la redondance. }\end{array}$ \\
\hline $\begin{array}{l}\text { Discussion en } \\
\text { sous-groupes } \\
\text { de 3-6 participants }\end{array}$ & $\begin{array}{l}\text { Elevé-moyen } \\
\text { si plus nombreux }\end{array}$ & $\begin{array}{l}\text { Effectuer une tâche commune } \\
\text { en fonction d'un résultat } \\
\text { attendu (A, B, C). }\end{array}$ & $\begin{array}{l}\text { Préciser le résultat attendu. } \\
\text { Donner des consignes claires sur } \\
\text { les tâches de chacun et la } \\
\text { démarche à suivre (incluant la } \\
\text { durée de chaque étape). }\end{array}$ \\
\hline $\begin{array}{l}\text { Démonstration } \\
\text { de techniques avec } \\
\text { consignes d'observation }\end{array}$ & Faible-moyen & $\begin{array}{l}\text { Démontrer une technique } \\
\text { comme modèle de rôle et faire } \\
\text { appel aux suggestions des par- } \\
\text { ticipants lors d'arrêts brefs (B). }\end{array}$ & $\begin{array}{l}\text { Préciser quoi observer dans la } \\
\text { démonstration pour garder le } \\
\text { focus sur ce qui est important } \\
\text { (fournir un outil écrit au } \\
\text { besoin). }\end{array}$ \\
\hline $\begin{array}{l}\text { Jeux de rôle et feedback: } \\
\text { - traditionnels }(\mathrm{B}, \mathrm{C}) \text {, } \\
\text { - collectifs }(\mathrm{B}, \mathrm{C}), \\
\text { - pratique en trios }(\mathrm{B})\end{array}$ & $\begin{array}{l}\text { Faible } \\
\text { Moyen } \\
\text { Elevé }\end{array}$ & $\begin{array}{l}\text { Expérimenter et améliorer une } \\
\text { habileté ou réfléchir sur des } \\
\text { attitudes }(\mathrm{B}, \mathrm{C}) \text {. }\end{array}$ & $\begin{array}{l}\text { Voir l'article de Girard et al. }{ }^{14} \\
\text { pour prévoir la planification et } \\
\text { l'animation des divers jeux de } \\
\text { rôle. }\end{array}$ \\
\hline $\begin{array}{l}\text { Retour en plénière : } \\
\text { important pour boucler } \\
\text { chaque exercice et discus- } \\
\text { sion. }\end{array}$ & Faible-moyen & $\begin{array}{l}\text { Effectuer des synthèses suite } \\
\text { aux exercices et discussions } \\
\text { afin de souligner les prises de } \\
\text { conscience ou les messages à } \\
\text { retenir }(A, B, C) \text {. }\end{array}$ & $\begin{array}{l}\text { Annoncer avant l'exercice ce } \\
\text { que vous ciblerez lors de la plé- } \\
\text { nière. Éviter la redondance en } \\
\text { choisissant la technique appro- } \\
\text { priée. }\end{array}$ \\
\hline \multicolumn{4}{|c|}{$\begin{array}{l}\text { *A : ateliers de production, } B: \text { ateliers d'apprentissage, } C \text { : ateliers d'exploration. } \\
\text { ** Idéalement, le contenu théorique nécessaire à l'atelier de type } B \text { est fourni au préalable aux participants qui l'ont lu } \\
\text { comme pré-requis à l'atelier. Alors, les animateurs accueillent les questions de clarification ou les questionnent brièvement } \\
\text { pour s'assurer de leur comprébension. Cela est plus réaliste dans un processus continu d'apprentissage qu'en éducation } \\
\text { médicale continue. }\end{array}$} \\
\hline
\end{tabular}




\section{Planification et animation de trois types d'ateliers pédagogiques}

\section{Guide sur la PRATIQUE EN TRIO}

Ce que c'est ? Les participants sont regroupés en trios où, dans trois mises en situation (scénarios fournis par écrit), chacun joue à tour de rôle, en rotation, les rôles d'interviewé ou de patient, d'interviewer ou de médecin et de collègue observateur à l'aide d'une grille d'observation indiquant les comportements attendus.

Déroulement typique : Les animateurs facilitent le déroulement en gérant le temps de façon stricte. Les participants lisent d'abord leur rôle (1-3 minutes). Ils procèdent ensuite au premier jeu de rôle et le terminent au signal des animateurs (5-7 minutes). L'observateur gère la période de feedback (4-5 minutes). Il demande d'abord aux protagonistes un commentaire, par exemple, sur ce qui a été difficile pour l'interviewer et ce qui a été aidant pour l'interviewé. Puis, il formule ses observations sous la forme d'un comportement à poursuivre et un élément à améliorer. Le tout est répété à deux reprises pour permettre à chacun d'expérimenter les trois rôles. L'exercice se termine par un retour en plénière.

\section{Règles de base des choix à faire pour le planificateur :}

\section{Faut-il démontrer d'abord ou faire pratiquer directement?}

Si l'habileté à pratiquer est simple à comprendre et proche de ce que les participants font déjà, il suffit de la fournir par écrit sous forme de grille d'observation détaillée et de l'expliquer sommairement avant l'exercice en faisant le lien avec les connaissances sous-jacentes.

Si l'habileté est complexe ou si la façon de procéder est- probablement- inhabituelle pour les participants, il est préférable d'effectuer d'abord une démonstration de ce qu'ils auront à faire en trio à partir d'une quatrième mise en situation et de répondre aux questions de clarification.

\section{La mise en situation écrite doit-elle être sommaire ou inclure des détails explicites sur la manière de réagir?}

Dans un groupe de 6 à 12 participants, au sein desquels les animateurs peuvent circuler dans les trios pendant l'exercice et donner un soutien concret à l'observateur dans la phase de retour sur l'action, le texte qui décrit les rôles d'interviewer et d'interviewé peut être sommaire. Il fournit les éléments de contexte nécessaires pour se mettre dans la peau du personnage et les énoncés ou réponses à formuler au départ tout en laissant beaucoup de place à l'improvisation. Dans un groupe où les participants sont plus nombreux, où les apprentissages doivent être soutenus entièrement par le matériel d'exercice fourni aux participants, il faut inclure des indications claires qui guident la façon de jouer les rôles pour que les interventions ainsi programmées donnent un feedback direct à l'interviewer. Il faut donc :

a) pour l'interviewer, décrire précisément la séquence des tâches à effectuer et indiquer des interventions clés à utiliser en présence de difficultés ;

b) pour l'interviewé, présenter des options de réponses à donner selon les diverses avenues que l'interviewer est susceptible d'emprunter ;

c) pour l'observateur, fournir une grille détaillée des comportements attendus et des correctifs à proposer dans l'éventualité d'écarts marqués par rapport aux attentes.

La préparation de ce type de matériel nécessite plus d'expérience pour être au point et conduire à un exercice d'apprentissage efficace.

\section{La composition des sous-groupes doit-elle être aléatoire ou planifiée?}

La composition des sous-groupes est généralement aléatoire dans un groupe homogène ; la distribution est laissée à la spontanéité des participants ou programmée (ex. : code de couleurs sur l'insigne du participant). Dans un groupe hétérogène comprenant à la fois des participants expérimentés et des novices ou différents professionnels, elle a avantage à être planifiée. Les gens sont alors répartis dans les divers trios de manière à s'assurer que les tâches soient réussies tout en mettant à profit la diversité des participants.

\section{Le retour en plénière doit-il porter sur le contenu ou sur le processus?}

Dans les groupes comportant plus de 12 participants, la plénière porte essentiellement sur le processus. Les participants commentent leurs réussites et leur progression de même que les difficultés rencontrées dans cette forme d'exercice. S'il est utile de revenir sur le contenu, ce sera sous la forme des messages à retenir en s’assurant de les clarifier si nécessaire à partir des questions de l'auditoire. Dans un groupe de 6 à 12 participants, les animateurs, à condition qu'ils aient assisté les observateurs dans leur rôle, pourront également revenir en plénière sur les erreurs de contenu les plus fréquentes et suggérer des façons d'y remédier, voire proposer un nouvel exercice pour compléter l'apprentissage. 


\section{Références}

à situer leur progression par rapport aux messages de l'atelier et aux apprentissages visés et ainsi faciliter grandement l'animation. La planification judicieuse et souple du temps dévolu à chaque activité est également facilitante. En cours d'atelier, les animateurs stimulent des interactions harmonieuses et gèrent efficacement la discussion par rapport aux objectifs de l'atelier ; ils mettent en évidence les progrès accomplis et ce qui reste à faire en fonction du temps disponible. Une des tâches premières de l'animation est d'allouer aux participants plus de temps de travail actif à résoudre des problèmes et à générer des solutions que de temps d'écoute des animateurs. Par ailleurs, ceux-ci aident le groupe en partageant brièvement leurs expériences professionnelles en tant que modèles de rôle. Steinert ${ }^{18,19}$ met en évidence que plus l'animation est structurée, discrète et centrée sur la création et le maintien d'un climat de travail agréable et productif, plus elle est efficace et appréciée.

En plus de leur rôle consistant à guider les exercices, les animateurs interviennent verbalement surtout pour faciliter les retours et effectuer les synthèses en lien avec les apprentissages visés et les messages de l'atelier. Pour éviter la redondance lors des plénières, les animateurs d'un atelier de production colligent successivement les idées émises par chaque sous-groupe de participants, en leur demandant à tour de rôle de les énoncer, une à la fois, et de mentionner seulement ce qui est différent de ce qui a déjà été formulé. Bien qu'ils n'aient pas le contrôle du contenu à gérer, ils doivent savoir l'utiliser en fonction des messages clés en le recadrant et le synthétisant au service de ces messages. Dans les plénières d'un atelier de formation, ils se centrent plutôt sur le processus que sur le contenu en demandant au groupe d'identifier ce qui a été le plus utile et/ ou difficile au cours des exercices. Au besoin, ils stimulent les participants silencieux pour qu'ils partagent la responsabilité du succès du groupe et contrôlent avec tact les interventions de certains participants qui divergent de l'atteinte des objectifs visés ${ }^{2}$.

\section{Grille de contrôle de qualité d'un atelier}

Obtenir des participants une évaluation individuelle écrite de l'atteinte des objectifs visés et de l'efficacité de l'animation permet aux animateurs d'améliorer les futurs ateliers et de s'améliorer dans leurs tâches. Outre une évaluation écrite, plusieurs demandent aux participants de mentionner en quelques mots ce qu'ils retiennent de l'atelier ou un changement concret qu'ils comptent effectuer suite à l'atelier. Il est utile de les inviter à s'engager par écrit, pour eux- mêmes, sur ce qu'ils comptent faire différemment à court terme à la suite à leur participation à l'atelier.

Les auteurs vous proposent d'utiliser une grille de contrôle de qualité (tableau 3) à la fois au moment de vérifier si votre atelier est prêt à être mis en œuvre et comme outil d'auto-évaluation après votre atelier. Vous pourriez aussi demander aux participants de la compléter pour formuler leur feedback sur votre atelier. Dans le tableau 3, nous avons aussi indiqué les erreurs courantes observées lors d'ateliers auxquels nous avons participé. Lorsqu'on utilise cette grille comme outil d'auto-évaluation ou de feedback, la colonne de droite est vierge au départ et sert à indiquer si la performance observée est conforme aux comportements souhaités et à formuler des suggestions pour améliorer une performance ultérieure.

Nous recommandons aux novices en planification et animation d'ateliers de consulter un expert dès le début du processus. La grille d'observation sera utile à l'expert lors de la mise à l'essai de l'atelier. Ne soyez pas étonné que votre atelier ne soit rodé qu'après les deux ou trois premières prestations, à la suite desquelles vous aurez intégré progressivement les ajustements requis.

\section{Conclusion}

Larticle présente les conditions précises de planification, d'animation et d'évaluation de trois types d'ateliers. Il insiste sur l'importance de bien définir les besoins auxquels l'atelier devra répondre sous forme, par exemple, de comportements différents ou nouveaux à adopter par les participants. Il stipule les règles de choix d'exercices stimulants et illustre quelques situations à cet effet. Il fournit un outil d'évaluation, sous forme d'une grille de contrôle de la qualité d'un atelier, incluant les erreurs fréquentes observées ainsi que des suggestions pour les corriger.

Les participants perçoivent rarement la somme d'énergie nécessaire à la préparation et à l'animation d'un atelier réussi. La planification d'un atelier, telle que présentée ici, demande de trois à cinq fois le temps que durera l'atelier lui-même. Par exemple, un animateur novice, préparant un atelier de deux heures, prévoira dix heures de planification ; un animateur chevronné parviendra à le faire en six heures.

L'animation d'un atelier requiert des habiletés intellectuelles de concentration, de synthèse et d'organisation, ainsi que des qualités d'adaptation au groupe qui requièrent des capacités affectives d'écoute, d'empathie, 


\section{Planification et animation de trois types d'ateliers pédagogiques}

de soutien et d'encouragement au groupe. En raison de la variété et de l'ampleur des tâches à accomplir, il est recommandé de travailler en co-animation pour partager l'énergie requise par la gestion de l'atelier; dans ce contexte, les deux personnes se partagent les préoccupations du processus et du contenu et peuvent alterner ces deux rôles selon les moments.

A la fin de l'atelier, les animateurs se rencontrent pour échanger à chaud sur le déroulement, les situations clés d'animation et noter deux ou trois améliorations souhaitées. Ils se rencontreront à nouveau quelques jours plus

\section{Références}

1. Jean P. Pour une planification méthodique des activités de formation. Pédagogie Médicale 2001;2:101-7.

2. Gutnik NL, Gutnik F. Réussir la conception et l'animation de formations. Paris: Vuibert, 2005.

3. Boulé R, Girard G. La résidence en médecine de famille : Difficultés et solutions. Can Fam Physician 2003;49:472-82.

4. Boulé R, Girard G. L'annonce d'une mauvaise nouvelle. Dans : Richard C, Lussier MT. La Communication professionnelle en santé. SaintLaurent(Québec) : ERPI 2005, chap.10:267-89.

5. Martineau B, Girard G. Guide du mentor. Intégration Clinique et Professionnelle II. Faculté de médecine et des sciences de la santé, Université de Sherbrooke, atelier 11 .

6. Boulé R, Girard G. L'intimité dans la relation patientmédecin. Le Clinicien 2003:115-23.

7. Delbecq AL, Van DE Ven AH, Gustafson DH. Group Techniques for program planing : a guide to nominal group and Delphi processes. Glenview, Ill : Scott Foresman ; 1975.

8. Puchta C, Potter J. Focus group practice. London : Sage Publications, 2004.

9. Rotem A, Manzie P. How to use small groups in medical education. Medical Teacher 1980;2:80-7. tard pour planifier la nouvelle édition de l'atelier en tenant compte des suggestions des participants et de leurs propres réflexions sur leur animation. Nous espérons que les outils proposés faciliteront cette démarche lorsque vous préparerez et animerez votre prochain atelier.

\section{Contributions}

Gilles Girard, Diane Clavet et Richard Boulé ont contribué collégialement et solidairement à la conception et à la rédaction du manuscrit.
10. Clavet D, Langlois L. Planifier et animer un atelier. Matériel d'atelier non publié (disponible sur demande auprès des auteurs). Sherbrooke, 2005.

11. Chamberland M, Hivon R. Les compétences de l'enseignant clinicien et le modèle de rôle en formation clinique. Pédagogie Médicale 2005;6:98-111.

12. Flanagan JC. The critical incident technique. Psychol Bull 1954;51:327-58.

13. Côté L, Leclère H. Pourquoi "se centrer sur le participant" en formation médicale continue? Pédagogie Médicale 2000;1:40-4.

14. Girard G, Clavet D, Boulé R. Planifier et animer un jeu de rôle profitable pour l'apprentissage. Pédagogie Médicale 2005;6:2-9.

15. Schön DA. Educating the reflexive practitioner : Toward a new design for teaching and learning in the professions. San Francisco : Jossey-Bass, 1987.

16. Boulé R, Girard G, Clavet D, Bernier C. Stratégies d'animation de groupe sur la communication patient-médecin-famille. Pédagogie Médicale 2000;1:13-22.

17. Tardif J. Le transfert des connaissances. Montréal : Les Editions Logiques, 1999.

18. Steinert $Y$. Twelve tips for conducting effective workshops. Medical Teacher 1992;14:127-31.

19. Steinert $Y$. Twelve tips for effective small-group teaching in the health professions. Medical Teacher 1996;18:203-7.

Manuscrit reçu le 8 novembre 2007; commentaires éditoriaux formulés aux auteurs le 21 décembre 2007; accepté pour publication le 31 décembre 2007. 